\title{
OLYMPUS
}

Your Vision, Our Future

\section{Visualizing axon guidance phenotypes induced by RNAi in chicken embryos}

The ability to turn off a gene of interest in a temporally and spatially controlled manner is key to the analysis of gene function in developing organisms. However, the induction of temporally controlled loss-of-function phenotype is very difficult. A combination of RNA interference (RNAi) with fluorescence microscopy analysis is proving to be a very good solution to this problem. Here we show that the Olympus SZX stereo and upright BX microscopes, both with fluorescence illumination capabilities, are perfect for use in these situations.

For decades the chicken embryo has been a classical model system for developmental studies in vertebrates because of its easy accessibility for in vivo studies. However, its major drawback for functional gene analysis has been the lack of tools to modify gene activity. Now, with the possibility of gene silencing by in ovo RNAi, the chicken embryo has once again re-emerged as an excellent vertebrate model system.

To study the results of the silencing efficiently requires the use of high-quality fluorescence imaging systems to visualize the effects at molecular, organ, system and whole-animal levels. To serve such cutting-edge research requirements, Olympus continuously improves its full range of fluorescence microscopes. The Olympus SZX stereo microscopes can be used to efficiently detect fluorescence signals in the whole organism and then zoom in on regions of interest. For investigations that require higher magnification—at cellular and subcellular level- the Olympus fluorescence research microscopes, such as the BX51 upright microscope, provide the resolution and sensitivity to detect even the finest details. As a result, visualization in functional gene analysis studies has become possible at both the subcellular and whole-organism levels.

\section{Gene silencing by in ovo RNAi}

Because of its easy accessibility, the chicken embryo has become a favored model system to study central nervous system development. One focus, for example, is on the molecular mechanisms of axonal pathfinding ${ }^{1-3}$, when developing commissural axons in the spinal cord cross the ventral midline and then the floor plate and subsequently turn rostrally along the longitudinal axis.

\section{Winfried Busch}

Olympus Life Science Europa, Microscopy, Wendenstrasse 14-18, D-20097 Hamburg, Germany. Correspondence should be addressed to W.B.

(microscopy@olympus-europa.com).
To determine the 'guidance cues' for this complex and essential process, Pekarik and collegues ${ }^{2}$ developed an RNAi-based assay to characterize the involvement of previously identified cDNA candidates. a
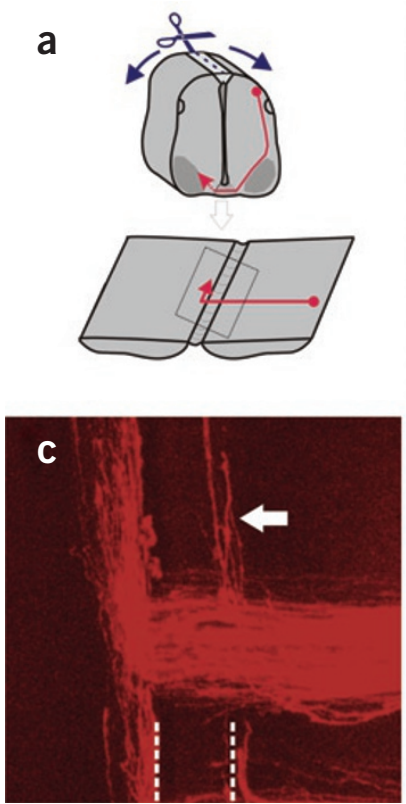

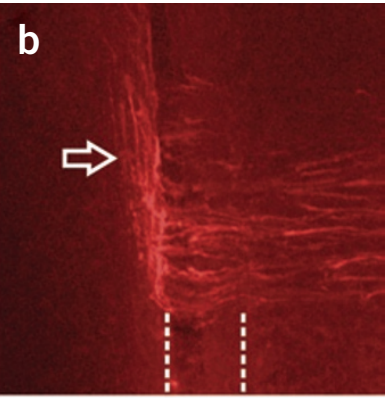

d

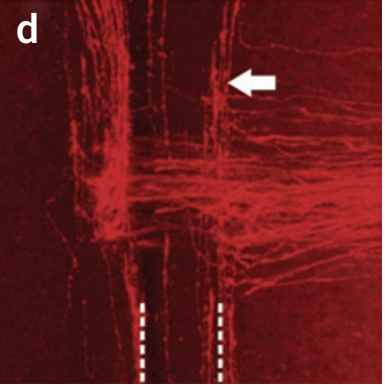

Figure $\mathbf{1}$ | Specific and efficient silencing of target genes by in ovo RNAi, visualized using an Olympus BX51 microscope equipped with fluorescence optics. (a) For the analysis of axonal pathfinding phenotypes, spinal cords are dissected and cut open at the dorsal midline. Micrographs of the boxed area are shown in $\mathbf{b}-\mathbf{d}$. (b) The trajectories of commissural axons can be traced with the lipophilic dye, Dil, that is applied to the cell bodies. In control embryos, commissural axons cross the floor plate (dashed lines) before they turn rostrally along the contralateral floor-plate border (open arrow). (c,d) When the function of the axon guidance cue axonin-1 is perturbed, either at the protein level by the injection of function-blocking antibodies (c) or by in ovo RNAi (d) commissural axons turn erroneously along the ipsilateral floor-plate border (arrow) and some do not cross the midline. Figure adapted from ref. 2 . 


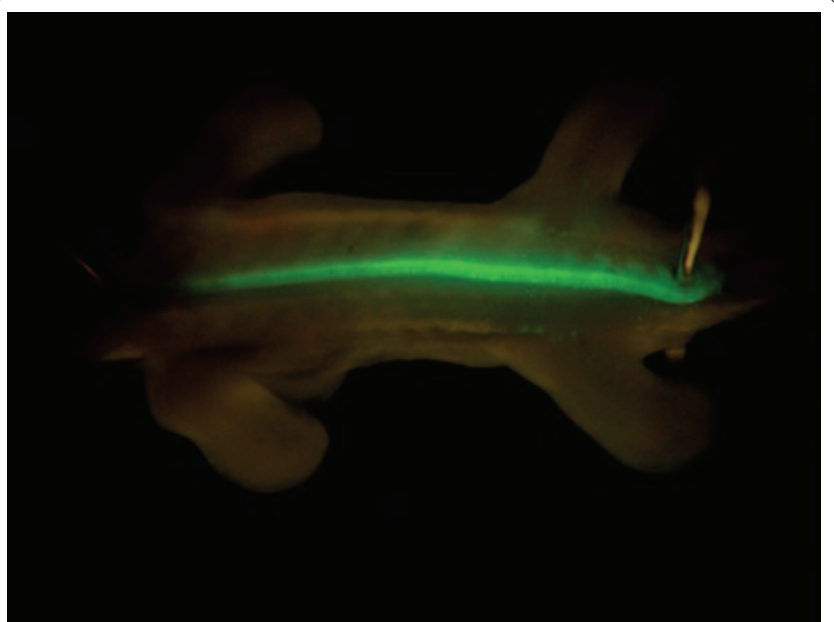

Figure $\mathbf{2} \mid$ In this 5-day-old chicken embryo imaged with an Olympus SZX12 stereo microscope equipped with fluorescence optics, GFP can easily be detected in the spinal cord. A plasmid encoding EGFP under the control of the $\beta$-actin promoter was electroporated into the embryo on the third day of incubation

A comparison of the loss-of-function phenotypes induced by functionblocking antibodies and by this in ovo RNAi process using fluorescence microscopy on an Olympus BX51 microscope showed that the results of the two techniques were comparable (Fig. 1).

\section{Visualizing the silence up close}

To visualize the effect of this gene-silencing experiment, an open-book preparation can be used. For this, the spinal cord is dissected out of the embryo, and the dorsal midline is cut to open the neural tube like a book (Fig. 1a). This allows the trajectory of groups of commissural axons to be visualized using fluorescent dyes such as Dil (which labels cell bodies) and an Olympus BX51 microscope ${ }^{4}$. The outstanding optical properties of the Olympus UIS2 system, especially the very high signal-to-noise ratio, permit detection of even the faintest signals in the specimen. In this experimental setup, a GFP transgene was used as a marker of electroporation efficiency. Clear imaging of GFP in this system enabled researchers to show that $60 \%$ of the cells in electroporated areas of the spinal cord expressed the transgene ${ }^{2}$ (Fig. 2).

\section{Visualizing the silence from afar}

It is also important to be able to visualize the effect of gene silencing on axonal guidance in whole-mount preparations, as this allows a broader view of the peripheral nervous system 'from macro to micro'. This is achieved by antibody labeling of the neurofilaments, the characteristic cytoskeletal elements of neurons, followed by staining with a fluorescent secondary antibody. The whole-organism view can

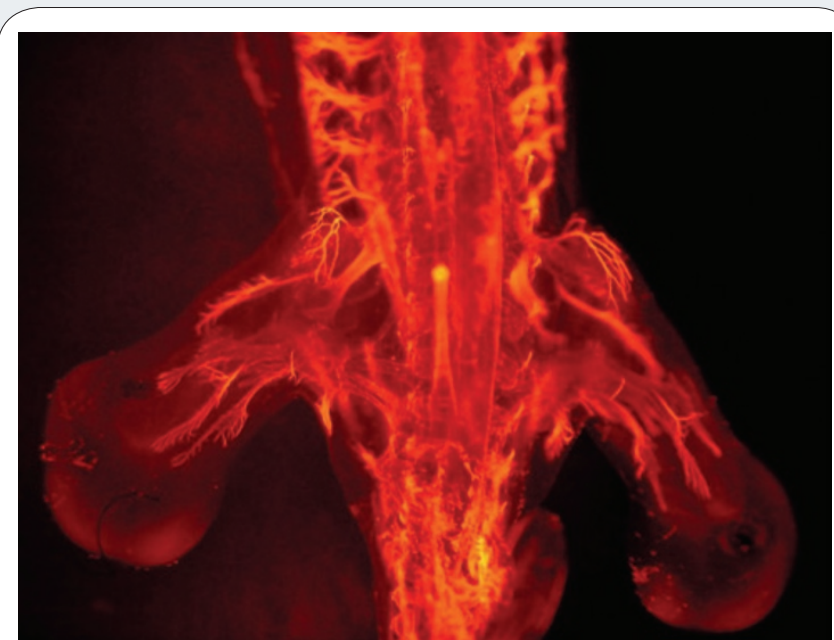

Figure $\mathbf{3}$ | Axonal pathfinding in the peripheral nervous system can be visualized by anti-neurofilament staining of intact embryos. Here an antibody recognizing the 160-kDa subunit of neurofilament was used followed by a Cy3labeled secondary antibody. Tissue was cleared in a graded series of methanol and imaged after transfer to a mixture of benzyl alcohol and benzyl benzoate using an Olympus SZX12 microscope.

then be generated on an advanced fluorescence-enabled stereo microscope, such as a microscope from the Olympus SZX series (Fig. 3) that was specifically developed to provide maximum possible resolution, clarity and zoom range.

\section{Conclusion}

The possibility of silencing genes in chicken embryos using in ovo RNAi makes the avian embryo an excellent model system for developmental studies and a powerful tool for functional genomics. The former limitations in genetic manipulation methods have been resolved by combining in ovo RNAi with electroporation and fluorescence microscopy-stereomicroscopy in the easily accessible chicken embryo.

\section{ACKNOWLEDGMENTS}

We acknowledge the assistance of E. Stoeckli in the development of this manuscript.

1. Perrin, F.E., Rathjen, F.G. \& Stoeckli, E.T. Distinct subpopulations of sensory afferents require F11 or axonin-1 for growth to their target layers within the spinal cord of the chick. Neuron 30, 707-723 (2001).

2. Pekarik, V. et al. Screening for gene function in chicken embryo using RNAi and electroporation. Nat. Biotechnol. 21, 93-96 (2003).

3. Bourikas, D. et al. Sonic hedgehog guides commissural axons along the longitudinal axis of the spinal cord. Nat. Neurosci. 8, 297-304 (2005).

4. Perrin, F.E. \& Stoeckli, E.T. Use of lipophilic dyes in studies of axonal pathfinding in vivo. Microsc. Res. Tech. 48, 25-31 (2000).

This article was submitted to Nature Methods by a commercial organization and has not been peer reviewed. Nature Methods takes no responsibility for the accuracy or otherwise of the information provided. 